Revue des patrimoines

46 | 2022

Le patrimoine de la Justice

\title{
Le fonds des dessins d'architecture du palais de justice aux Archives de Paris
}

The collection of architectural drawings of the palais de justice in the Archives of Paris

\section{Nicolas Courtin}

\section{(2) OpenEdition}

Journals

Édition électronique

URL : https://journals.openedition.org/insitu/33908

DOI : 10.4000/insitu.33908

ISSN : 1630-7305

Éditeur

Ministère de la Culture

Référence électronique

Nicolas Courtin, «Le fonds des dessins d'architecture du palais de justice aux Archives de Paris », In Situ [En ligne], 46 | 2022, mis en ligne le 16 janvier 2022, consulté le 03 février 2022. URL : http:// journals.openedition.org/insitu/33908; DOI : https://doi.org/10.4000/insitu.33908

Ce document a été généré automatiquement le 3 février 2022.

In Situ Revues des patrimoines est mis à disposition selon les termes de la licence Creative Commons Attribution - Pas d'Utilisation Commerciale - Pas de Modification 4.0 International. 


\section{Le fonds des dessins d'architecture du palais de justice aux Archives de Paris}

The collection of architectural drawings of the palais de justice in the Archives of Paris

Nicolas Courtin

1 En 2001, les Archives de Paris ont pris en charge un versement d'archives du tribunal de grande instance de Paris constitué de pièces graphiques jusque-là conservées par le service des Travaux de cette juridiction. Ce fonds se composait d'environ 270 portefeuilles contenant chacun entre 10 et 40 dessins, soit un total estimé alors autour de 10000 pièces. Ce versement considérable était l'aboutissement d'une prise de conscience de l'intérêt patrimonial de ce fonds entreprise par le tribunal depuis la fin des années 1980, principalement à l'instigation de Jean Favard (1934-2010), conseiller à la Cour de cassation. C'est à cette faveur que le tribunal a effectué les premiers récolements en 1989 et 1992, puis des études plus poussées ont été commandées au laboratoire IPRAUS ${ }^{1}$ (Institut parisien de recherche Architecture, Urbanistique et Société ; 1995) et au GRAHAL ${ }^{2}$ (Groupe de recherche Art, Histoire, Architecture, Littérature ; 1997). Une exposition organisée par l'Action artistique de la Ville de Paris en 2002 à la Conciergerie, accompagnée d'un catalogue scientifique ${ }^{3}$, marqua l'aboutissement de ce processus d'identification et de reconnaissance de la valeur du fonds.

2 Aux Archives de Paris, un traitement fut entrepris en 2002 mais est resté inachevé. Un instrument de recherche provisoire, compilant les inventaires préexistants et un début d'inventaire synthétique ont été mis à disposition des lecteurs. Parallèlement, l'absence de reconditionnement des plans rendait la communication en salle de lecture presque impossible. Une sélection de "pièces remarquables " a toutefois été faite, en accord avec le tribunal, et a permis la mise en ligne sur le site internet des Archives de Paris d'une exposition virtuelle présentant quelques feuilles $\mathrm{du}$ fonds ${ }^{4}$. 
3 À la demande de l'architecte en chef des monuments historiques ayant la charge du Palais, Christophe Bottineau, les Archives de Paris ont entrepris en 2016 le reconditionnement et le récolement du fonds. Depuis 2018, un instrument de recherche à jour est désormais disponible ${ }^{5}$ et une connaissance plus fine du fonds a été acquise.

\section{Un fonds plus riche que prévu}

4 Après cette opération, il a été possible de préciser la composition et la nature du fonds. Il comporte en réalité environ 15300 pièces - soit un accroissement de plus de $50 \%$ de l'estimation initiale - regroupées en 320 articles (et non pas 464 comme le premier instrument de recherche l'affirmait, ou 270 comme indiqué lors de la convention de versement de 2001). De natures et de formats très variés, les pièces sont très majoritairement des dessins mais le fonds comporte également des gravures et des tirages photographiques (moins d'une centaine).

5 Il s'agit donc d'un fonds de dessins d'architecture dans lequel domine largement la production manuscrite - les tirages imprimés sont relativement rares et concernent soit les plans généraux destinés à la publication, soit le chantier du bâtiment du tribunal correctionnel. On rencontre ainsi tous les types de supports et de techniques $\mathrm{du}$ dessin d'architecture du xIx siècle : crayon, plume et lavis, voire aquarelle, sur papier fort ou calque, papier huilé ou entoilé, et calque collé sur papier ou carton. La nature des dessins fournis par l'agence relevant de toutes les étapes de la création architecturale, les échelles - et par conséquent les formats - sont très variés. Du simple croquis sur un coin de feuille au dessin grandeur nature d'un meuble ou d'un chapiteau, le fonds comporte une multitude de formats, ce qui rend leur conservation dans des cartons à dessin de format $\mathrm{A} 0$ plus ou moins adaptée. Cette grande disparité, l'usage ancien de les conserver dans des portefeuilles inadéquats et les mauvaises conditions de conservation dans les locaux du Palais expliquent que l'ensemble soit aujourd'hui globalement en mauvais état - voire en très mauvais état en ce qui concerne les calques, pliés ou collés de longue date.

6 Le spectre chronologique s'est également vu modifié après le récolement de 2018. Annoncé par l'inventaire de 2002 comme couvrant un siècle, entre 1830 et 1930, le fonds s'avère plus riche, avec des pièces datées entre 1763 et 1966. Si l'ancienneté du fonds - due à la présence d'une gravure vénitienne du XVIII ${ }^{\mathrm{e}}$ siècle $^{6}$ - résulte du travail de documentation des architectes $\mathrm{du} \mathrm{xIX}^{\mathrm{e}}$ siècle et ne reflète pas les travaux conduits au Palais sous le règne de Louis XV, la date extrême des années 1960 indique bien des travaux effectués jusqu'au milieu du $\mathrm{xx}^{\mathrm{e}}$ siècle ${ }^{7}$. Néanmoins, cette amplitude plus large que prévu ne doit pas dissimuler que la majorité du fonds (environ 12470 dessins) se focalise sur la période 1838-1915, soit les deux grandes étapes de l'histoire du palais contemporain: le "projet d'agrandissement et d'isolement» décidé sous la Restauration et entrepris par la monarchie de Juillet, et la reconstruction quasi complète de l'édifice après 1871. Il s'agit donc bien d'un fonds « XIX siècle » auquel on doit apporter la connaissance du métier d'architecte de cette époque.

7 Tout en respectant le cadre de classement du fonds - qui conjugue l'organisation topographique et celle des services - le récolement a permis de mieux identifier les secteurs du Palais qui ont fait l'objet de travaux ${ }^{8}$. Les trois quarts du fonds concernent les principaux occupants du bâtiment: le tribunal civil et le tribunal correctionnel 
(36\%), la préfecture de police et la cour d'appel (24\%) et enfin la cour d'assises et le dépôt (16\%). Il est donc à noter une sous-représentation de la Cour de cassation, pourtant partie importante de la cité judiciaire du XIX ${ }^{\mathrm{e}}$ siècle.

8 Le quart restant concerne les plans généraux du bâtiment, les espaces communs et les abords, mais aussi des édifices autres que le palais de justice. Bien qu'anecdotique par rapport à l'ensemble du fonds ( $1 \%$ des pièces), ces dessins reflètent la diversité des activités des architectes ayant eu la charge du Palais. Il peut s'agir d'autres édifices de justice ou de police - comme le tribunal de commerce ou des travaux au ministère de la Justice, place Vendôme ${ }^{9}$ - ou des projets sans rapport direct, comme le Palais royal de Bruxelles ${ }^{10}$ (Honoré Daumet, 1898) ou l'université de Caen ${ }^{11}$ (Henry Bernard et Édouard Hur architectes, 1947), que les architectes semblent avoir égarés dans les cartons de l'agence. S'il y a des dessins d'édifices inattendus, il y a également des absences remarquables. Il est en effet à noter que l'agence d'architecture du Palais, palais national, n'avait pas dans ses attributions la Sainte-Chapelle, qui relevait de l'administration des Monuments historiques depuis 1830. Le fonds des Archives de Paris n'aborde donc qu'indirectement le joyau médiéval de la Cité ${ }^{12}$.

Comme la relative discrétion de la Cour de cassation l'a déjà suggéré, la composition du fonds reflète l'organisation administrative de la justice parisienne. Si le versement provient d'une même entité, le service des Travaux du tribunal de la Seine, les documents ont été produits par différents architectes. Si l'on devine une agence principale, il y a également des architectes attachés aux différents services concernés ${ }^{13}$. Aussi le fonds s'organise-t-il par secteurs et par maîtrises d'œuvre et, par conséquent, il se compose par zones et est peu transversal. Ainsi, le corpus de plans d'ensemble et de documents concernant les espaces communs est-il relativement modeste. À l'inverse, le dernier chantier d'importance mené au Palais, la construction de l'extension du tribunal civil par Albert Tournaire, quai des Orfèvres, est surreprésenté ( $15 \%$ du fonds). Cela peut s'expliquer par la relative "jeunesse » du chantier mais aussi par la maîtrise d'ouvrage qui a été aussi le service versant du fonds d'archives.

10 Ainsi le fonds apparaît-il plus riche qu'attendu en pièces et en contenu. Il se constitue autour d'un noyau fort qui est la conception et la mise en œuvre du projet de la Restauration d'une cité judiciaire moderne et organique, vaste et isolée, au cœur de la capitale. Mais l'histoire et les particularités administratives et judiciaires ont façonné différemment le cours du chantier et ont étiré les délais jusqu'à la veille de la Première Guerre mondiale, tout en occultant plus ou moins certaines parties de l'édifice. Le panorama est donc à la fois plus long que prévu mais aussi plus aléatoire. Enfin, l'analyse plus détaillée du fonds a mis en lumière la spécificité d'un fonds d'agence d'architecture, organisme qui collecte de la documentation aux fins des travaux à réaliser, produit toute la gamme des dessins nécessaires à la mise en place du projet, sa validation et son exécution mais qui, également, «oublie » dans les cartons des projets pour d'autres maitrises d'œuvre.

\section{Un chantier du XIX ${ }^{e}$ siècle}

11 De 1840 à 1960, le palais de justice de Paris fait continuellement l'objet de travaux. D'importance et de nature variables, leur organisation dans le temps et l'espace a été établie par Werner Szambien et Simona Talenti en 1995 précisément à partir de ces 
dessins ${ }^{14}$. Dresser le portrait de ce fonds, c'est donc aussi revenir sur la chronologie générale des interventions architecturales.

12 Au lendemain des incendies qui ont ravagé le Palais en 1737 et 1776 et de la Révolution, le grand principe des premiers projets du $\mathrm{XIX}^{\mathrm{e}}$ siècle est double: d'une part, dédier l'édifice exclusivement à la justice (avec ses différentes composantes: tribunaux, parquet, police...), et d'autre part, lui assurer les conditions de fonctionnement et de sécurité nécessaires. Architecturalement, cela se traduit par l'ambition d'agrandir les bâtiments et de les isoler physiquement des constructions environnantes, notamment dans la zone sud, où subsiste un groupe d'immeubles d'habitation à l'angle du quai des Orfèvres et de la rue de la Barillerie (emplacement de l'actuel boulevard du Palais).

\section{Le projet d'ensemble}

13 Les grandes lignes de ce projet ont été formalisées par l'architecte Jean-Nicolas Huyot (1780-1840) avant 1840. Mais n'ayant pu assurer sa mise en œuvre, ces plans échappent presque entièrement au fonds conservé aux Archives de Paris ${ }^{15}$. Celui-ci s'ouvre avec le perfectionnement du dessein de Huyot par les deux nouveaux architectes chargés du projet "d'agrandissement et d'isolement du Palais» à partir de 1840, Louis Duc (1802-1879) et Théodore Dommey (1801-1872). Cette date coïncide par ailleurs avec la mise en place du conseil des Bâtiments civils; il est aisé de deviner que l'existence de ce fonds a été motivé par la présentation régulière des projets à cette instance ${ }^{16}$.

L'examen des plans d'ensemble du Palais, entre les relevés de l'état avant 1840 et ceux dressés à la veille de la Première Guerre mondiale, alors que le bâtiment a atteint son extension maximum, rend compte des ambitions du projet de la Restauration. Un relevé de $1838^{17}$ fait état de l'imbrication des édifices d'origine médiévale et de ceux de l'époque moderne. Dans une sorte de conglomérat sans plan d'ensemble, on devine toutefois un début d'organisation rationnelle autour de la cour du Mai avec les travaux conduits au XVII ${ }^{\mathrm{e}}$ siècle pour relier entre eux le grand perron, la grande salle, la SainteChapelle et le logis du roi. Mais au-delà, c'est l'irrégularité des constructions dépendant de l'hôtel du Premier président ou de la Cour des comptes, qui se sont élevées sur une trame parcellaire épousant la topographie en trapèze de la pointe de l'île avant de rejoindre la barre d'immeubles de la place Dauphine ${ }^{18}$.

En 1903, [fig. 1] le Palais apparaît au contraire comme un bloc presque carré, isolé de la ville sur toute sa périphérie et avec une organisation intérieure clairement tramée par un système de cours et de galeries perpendiculaires qui délimitent des zones pouvant fonctionner indépendamment. L'adjonction, à l'angle du boulevard du Palais et du quai des Orfèvres, d'un dernier corps de bâtiment organisé autour d'une cour rectangulaire en 1910-1915 prolonge ce système. Ce faisant, l'architecte du Palais à la veille de la Première Guerre, Albert Tournaire, parachève le parti d'agrandissement, d'isolement et de rationalisation du bâtiment mis en place par Duc et Dommey. 
Figure 1

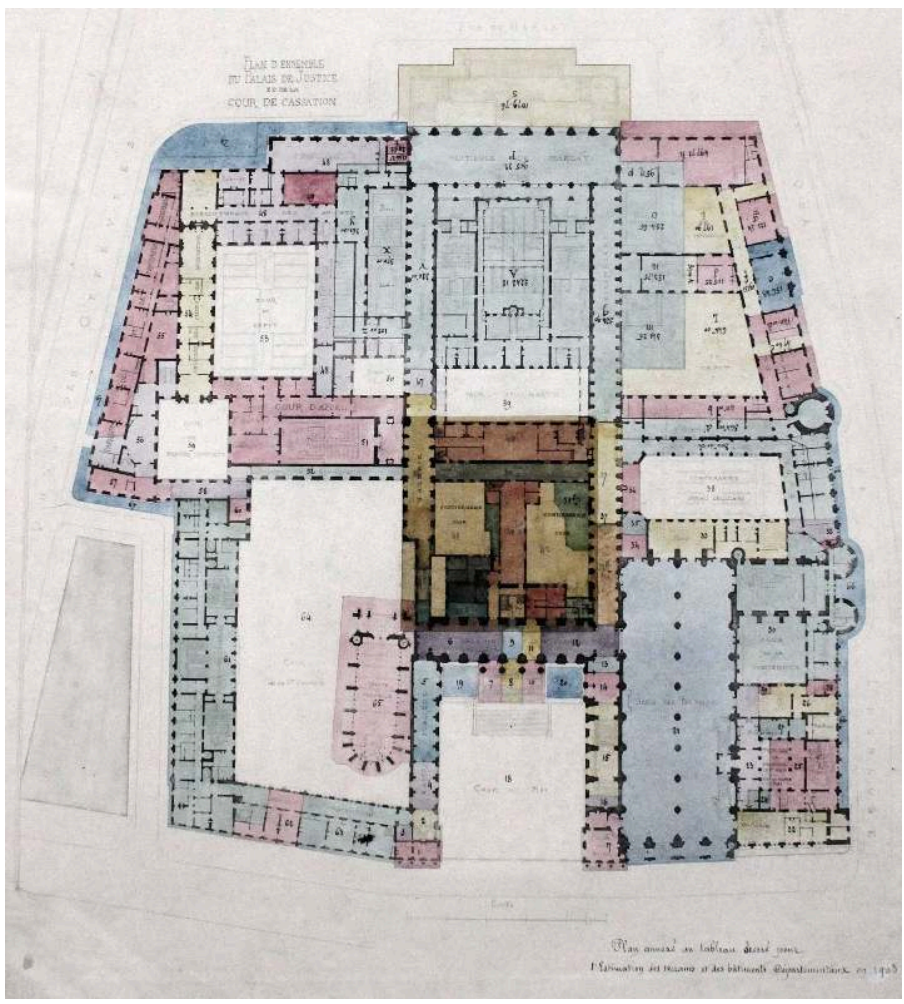

Plan d'ensemble du palais de justice et de la Cour de cassation. Plan imprimé rehaussé de couleurs, anonyme, 1903, conservé aux Archives de Paris (37Fi 3).

Reproduction Nicolas Courtin.

Ce parti avait été arrêté par les deux architectes en 1847 et est illustré dans notre fonds par un plan daté de $1849^{19}$ [fig. 2]. Celui-ci fait état très clairement de la position des architectes sur la question des édifices anciens. Dès cette date, il n'est prévu de conserver que les parties suivantes du Palais : la Sainte-Chapelle, les bâtiments bordant la cour du Mai, la grande salle et la Conciergerie, les quatre tours sur le quai de l'Horloge et les bâtiments autour de la cour de la Conciergerie et enfin, une partie du bâtiment de la Cour des comptes. 
Figure 2

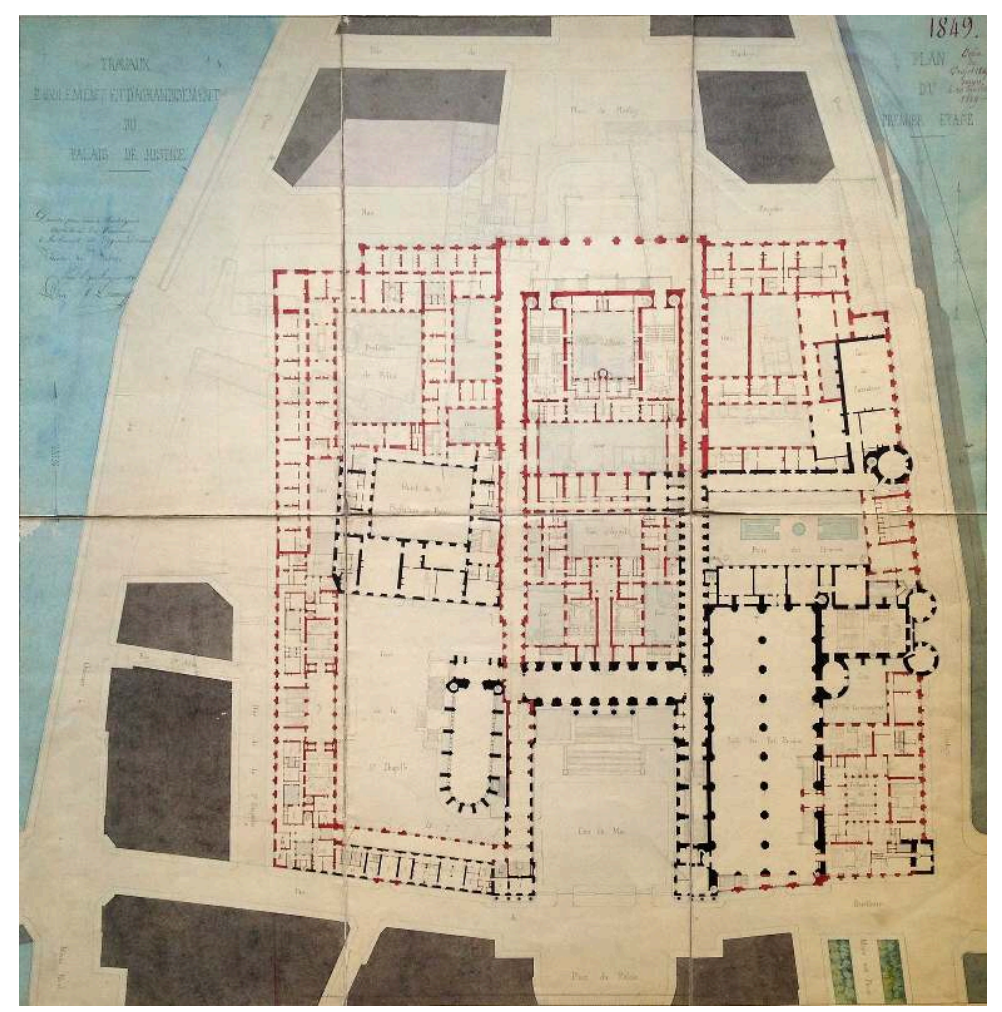

Travaux d'isolement et d'agrandissement du palais de justice. Plan du 7er étage. Copie du projet [de] 1847 [...]. Dessin aquarellé, dressé par Louis Duc et Théodore Dommey, 1849, conservé aux Archives de Paris (37Fi 12).

Reproduction Nicolas Courtin.

17 Le choix semble assez clair : conserver à la fois les hauts lieux de l'histoire du Palais Sainte-Chapelle, Grand'chambre, salle des pas perdus, prison révolutionnaire et bâtiment de Gabriel $^{20}$-, les amorces du projet moderne: la trame orthogonale de distribution - galerie Dauphine et aile Lamoignon - et naturellement, les constructions les plus récentes (bâtiment de l'Instruction).

\section{Les premiers travaux de Duc et Dommey}

Dès 1840, Duc et Dommey interviennent sur deux secteurs simultanément : le bâtiment de l'instruction et les chambres du tribunal civil. Rue de la Barillerie, au sud de la cour du Mai, le bâtiment de l'instruction est épaissi côté cour afin de créer une meilleure liaison avec le bâtiment projeté pour la police correctionnelle, plus au sud. Le corps de bâtiment construit sous Louis XVI reçoit ainsi une nouvelle façade sur la cour de la Sainte-Chapelle. Les relevés alors effectués par l'agence offrent un témoignage précieux et inédit sur l'architecture du XVIII ${ }^{\mathrm{e}}$ siècle [fig. 3]. 
Figure 3

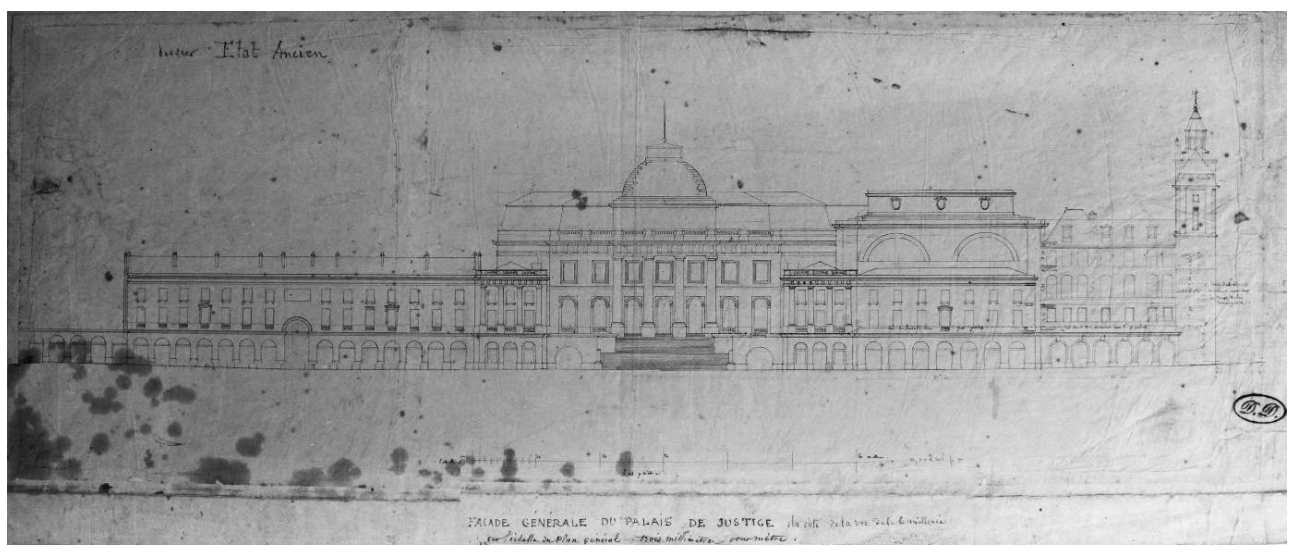

Façade générale du palais de justice du côté de la rue de la Barillerie. État ancien, Louis Duc et Théodore Dommey, dessin à plume, relevé vers 1849, conservé aux Archives de Paris (37Fi).

Reproduction Nicolas Courtin.

$19 \mathrm{Au}$ nord, Duc et Dommey entreprennent la démolition partielle des constructions hétéroclites de l'époque moderne qui occupent l'espace entre la grande salle, la tour de l'Horloge et le quai, en vue d'élever le bâtiment dit des «six chambres civiles » du tribunal de la Seine. Les premiers projets signés par eux datent de 1839 [fig. 4], et l'édification s'étend de 1849 à 1859. Les plans montrent un bâtiment à caractère double. $\mathrm{Au}$ rez-de-chaussée et à l'extérieur, les architectes ont composé avec les vestiges médiévaux emblématiques conservés - cuisine dite de saint Louis, structures de la Conciergerie et tour de l'Horloge, et créé les façades actuelles néogothiques, extrapolées de la tour d'angle. Parallèlement, à l'intérieur, ils ont conçu, dans une trame de maçonneries remployées, une distribution organisée autour d'un patio couvert néo-Renaissance, sans doute pour prolonger harmonieusement la grande salle de Salomon de Brosse qui en commande l'accès. 
Figure 4

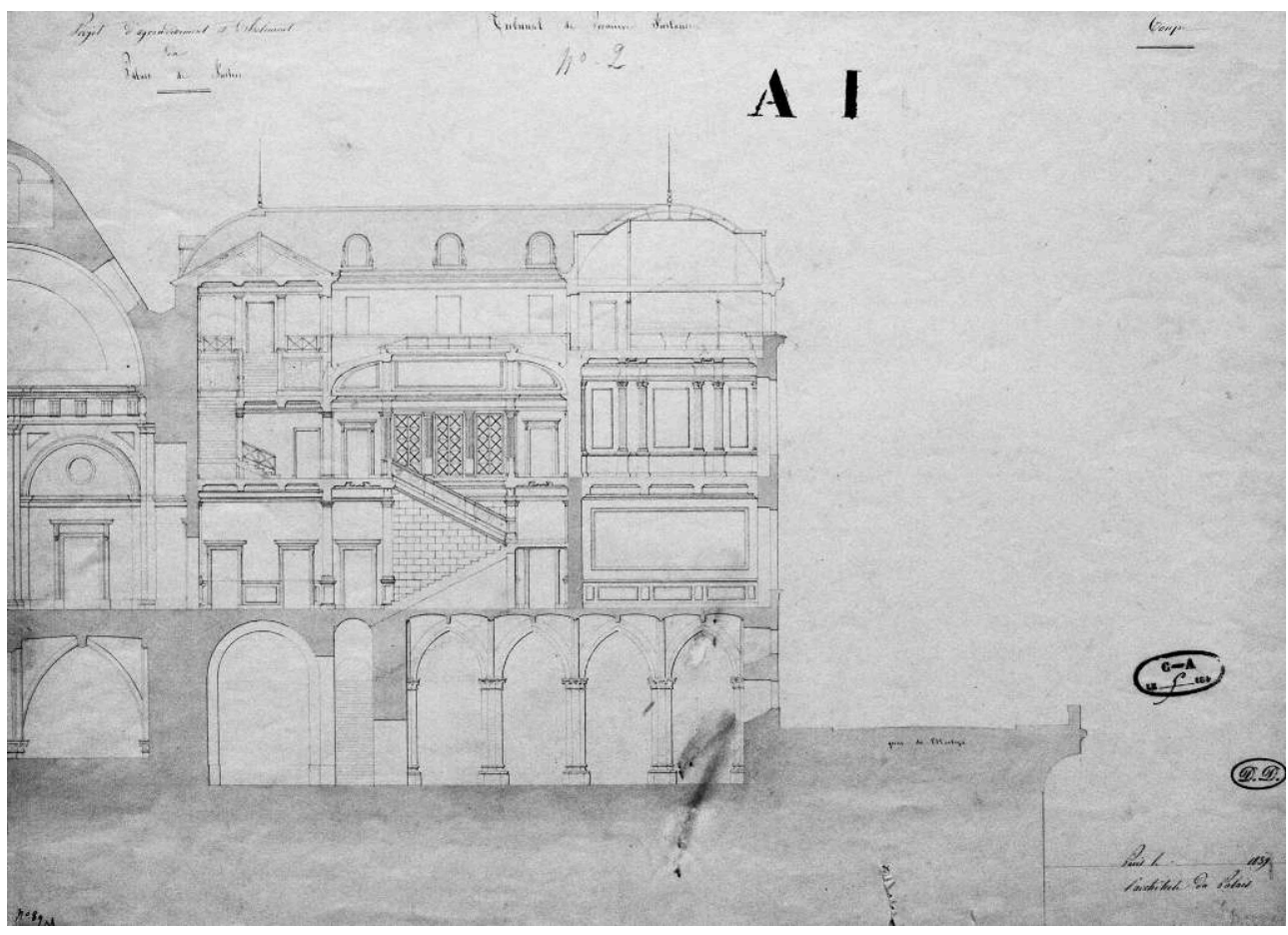

Projet d'agrandissement et d'isolement du palais de justice. Tribunal de première instance. Coupe, Louis Duc et Théodore Dommey, dessin, encre et lavis, 1839, conservé aux Archives de Paris (37Fi 224).

Reproduction Nicolas Courtin.

En complément de ce chantier, les deux architectes entreprennent la reconstruction du pignon de la grande salle sur la rue de la Barillerie (1850-1852). Motivée par la mise à l'alignement de la rue de la Barillerie, futur boulevard du Palais, cette opération entre parfaitement dans le projet de modernisation et d'isolement du palais puisqu'elle est l'occasion de démolir des immeubles placards du XviII siècle et de donner ainsi un nouveau frontispice à la salle des pas perdus (ainsi qu'un accès supplémentaire). Les feuilles du fonds documentent assez précisément les édifices à démolir [fig. 5] et les hésitations de Duc et Dommey quant au dessin de détail de la nouvelle façade remplages des baies thermales, silhouette des pignons... - en revanche, ils ne font état d'aucune hésitation sur le style architectural retenu : il s'est toujours agi d'élever une façade classique, à la manière de Salomon de Brosse, intercalée entre le pavillon néoclassique de Pierre Desmaisons à gauche et leur propre façade néogothique à droite. 
Figure 5

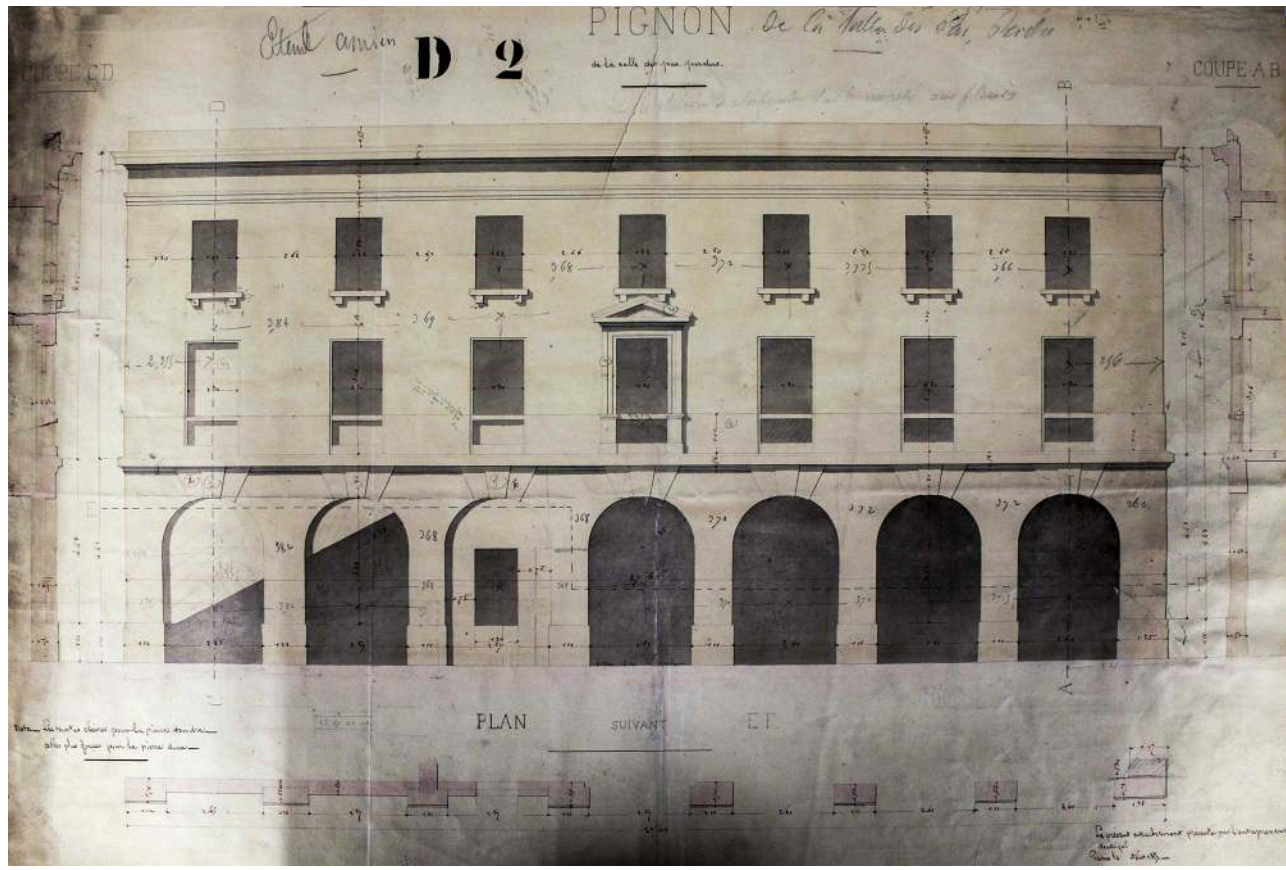

Pignon de la salle des pas-perdus. État ancien, dessin, encre et lavis, août 1852, conservé aux Archives de Paris (37Fi).

Reproduction Nicolas Courtin.

\section{Le bâtiment des Assises et du dépôt}

Dès 1852 est entreprise la partie sans doute la plus importante du projet de Duc et Dommey, le bâtiment des Assises et du dépôt. L'ensemble des plans concernant cette zone du Palais est particulièrement riche. Il offre de manière exemplaire toute l'étendue du travail de l'agence d'architecture du Second Empire à travers les étapes de la conception et de la construction d'un bâtiment neuf à l'emplacement de constructions anciennes. L'édifice, destiné à accueillir au rez-de-chaussée le dépôt des prisonniers et à l'étage les salles de la cour d'assises, doit également former le nouveau frontispice du palais du côté de la place Dauphine - au détriment des immeubles du $\mathrm{XvII}^{\mathrm{e}}$ siècle de la rue de Harlay. Son édification suppose d'importantes démolitions que le fonds documente à travers des relevés, des plans masses du secteur, des plans parcellaires et quelques photographies. Mais ce dossier s'enrichit également de dessins reflétant la réflexion stylistique des architectes jusqu'au choix définitif du style du vestibule et de la nouvelle façade [fig. 6]. Celle-ci, qui a fait l'objet de tous les soins de Duc et Dommey, est documentée jusque dans les croquis de détail de la sculpture monumentale et par une série de photographies réalisées le chantier à peine achevé, en 1868. 


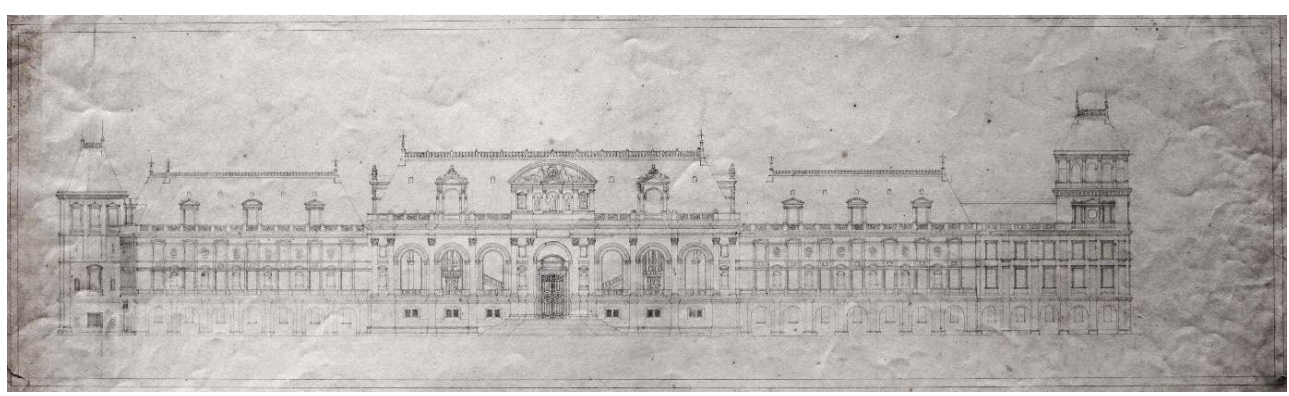

Bâtiment des Assises et du dépôt. Projet d'élévation rue de Harlay, dessin anonyme, vers 1850, conservé aux Archives de Paris (37Fi 166).

Reproduction Nicolas Courtin.

\section{La Cour de cassation}

Parallèlement au chantier des Assises et du dépôt, la Cour de cassation entreprend la reconstruction de son bâtiment. Le projet en est dressé vers 1856-1857 par Louis Lenormand (1801-1862), l'architecte de cette juridiction de 1849 à 1862. Il s'agit du secteur nord-ouest du Palais, adossé au bâtiment neuf de Duc et Dommey faisant suite au tribunal civil sur le quai de l'Horloge. Il est clair que dès les premiers projets, la volonté architecturale était de se distinguer des édifices avoisinants, néogothique d'un côté et moderne de l'autre, en choisissant le parti d'une architecture d'inspiration classique, parfaitement intégrée au paysage de bord de Seine, évoquant assez directement le Louvre. La nomination en 1862 de Louis Duc comme architecte de la Cour de cassation (jusqu'en 1879) permit sans doute de mieux intégrer l'édifice au palais. Ainsi, la façade en retour sur la place Dauphine adopte-t-elle finalement le dessin du bâtiment des Assises, et l'élévation de la façade au-delà de la tour médiévale reprend celle conçue par Duc et Dommey pour le bâtiment des chambres civiles.

\section{La restauration des parties médiévales}

L'espace situé entre la Cour de cassation et les chambres civiles correspond à la cour de la Conciergerie. Selon le projet de Duc et Dommey, elle fonctionne avec le dépôt (dont elle est séparée par la galerie Lamoignon), dont elle constitue la section réservée aux femmes. À l'intérieur des structures médiévales conservées, un important chantier de restauration est conduit entre 1859 et 1870 . Cette partie du fonds met notamment en lumière le travail extrêmement précis de re-création du second œuvre de style médiéval - menuiseries, vitrages, etc. [fig. 7]. 
Figure 7

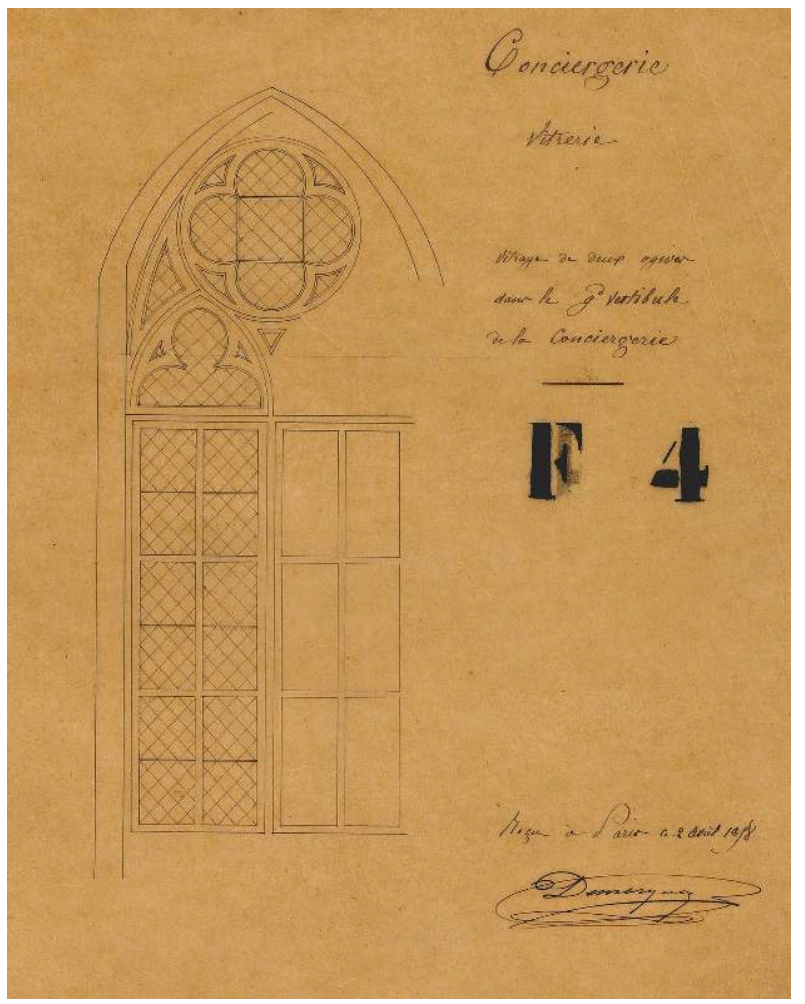

Vitrage de deux ogives dans le vestibule de la Conciergerie, Théodore Dommey, dessin à la plume, 1858, conservé aux Archives de Paris (37Fi 31).

Reproduction Nicolas Courtin.

Ce chantier accompagne celui de la restauration de la salle des pas perdus, qui est conduit dans les mêmes années 1860 . Le projet, désigné sur les plans comme une simple "appropriation» de la grande salle concerne certes des aménagements mobiliers modestes mais surtout, le renforcement des voûtes lourdement endommagées par l'incendie de 1776. C'est à cette occasion que sont posés des cintres métalliques destinés à soutenir les arcs doubleaux des berceaux de la salle, pourtant restaurés en 1782 par Antoine [fig. 8]. 


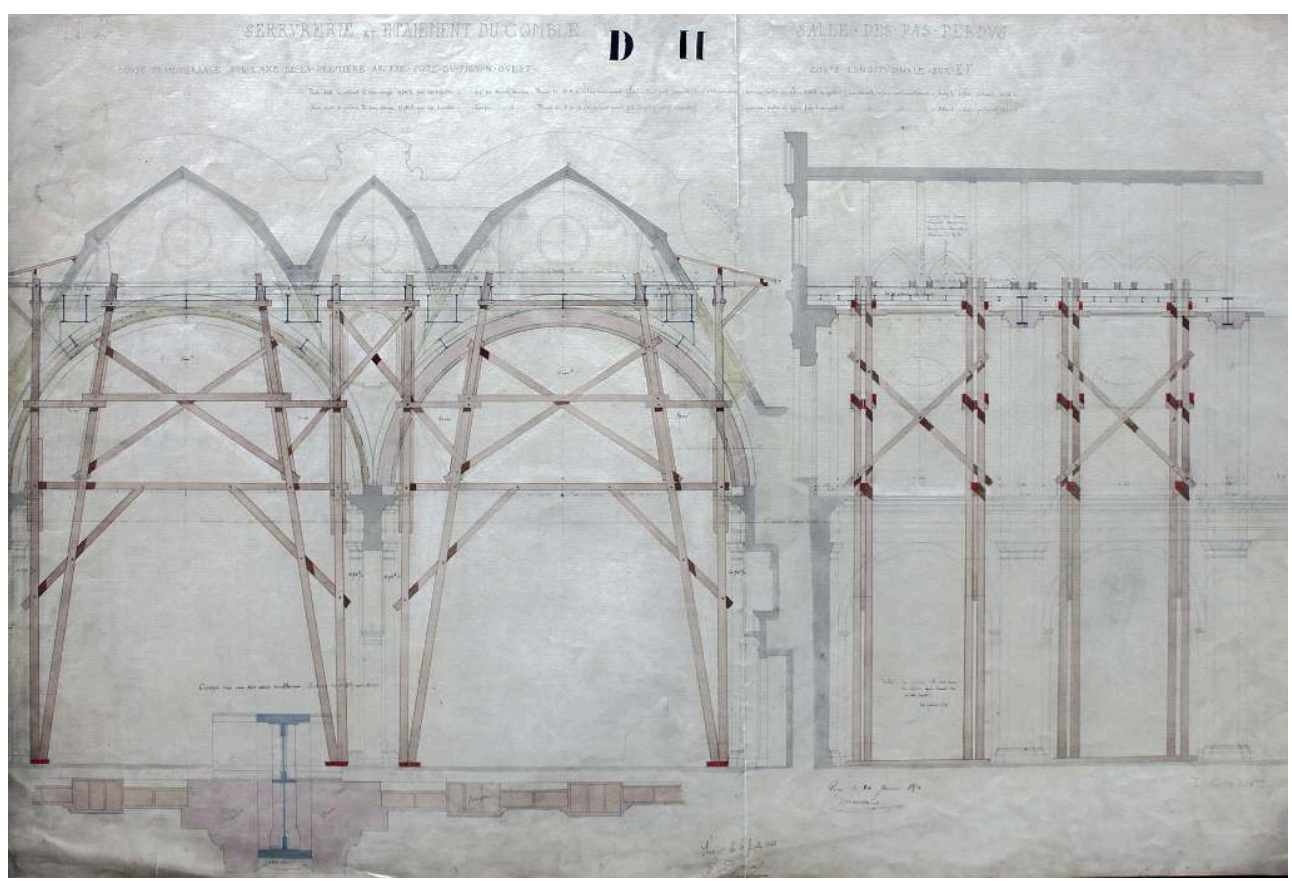

Serrurerie et étaiement du comble de la salle des pas-perdus [...], dessin, lavis encre et aquarelle, 1868, conservé aux Archives de Paris (37Fi 36).

Reproduction Nicolas Courtin.

\section{La préfecture de police}

À partir de 1855 commence la réflexion sur le réaménagement des locaux de la préfecture de police. Jusque-là installée dans l'ancien hôtel du Premier président (adossé à l'ouest du bâtiment de la Cour des comptes), accessible par la rue de Jérusalem (quai des Orfèvres), il fut d'abord envisagé, en 1855, la construction d'un bâtiment neuf à l'emplacement de la place Dauphine. Dès avant 1857, le choix était fait d'une reconstruction à la place de l'hôtel du Premier président. Cette nouvelle partie du Palais achevait de le régulariser en fermant l'angle sud-ouest du périmètre. Il formait une nouvelle façade sur le quai des Orfèvres et fonctionnait mieux avec le nouveau bâtiment des Assises et du dépôt. À l'instar du bâtiment de la Cour de cassation, cette nouvelle construction échappa à Duc et Dommey puisqu'elle fut conçue par les architectes de la préfecture Émile Gilbert (1793-1874) et Arthur Diet (1825-1890). Après avoir hésité avec un style "à la française ", ils proposèrent un édifice d'inspiration première Renaissance italienne d'une très grande qualité de dessin que rendent parfaitement les multiples croquis et calques du fonds [fig. 9]. 


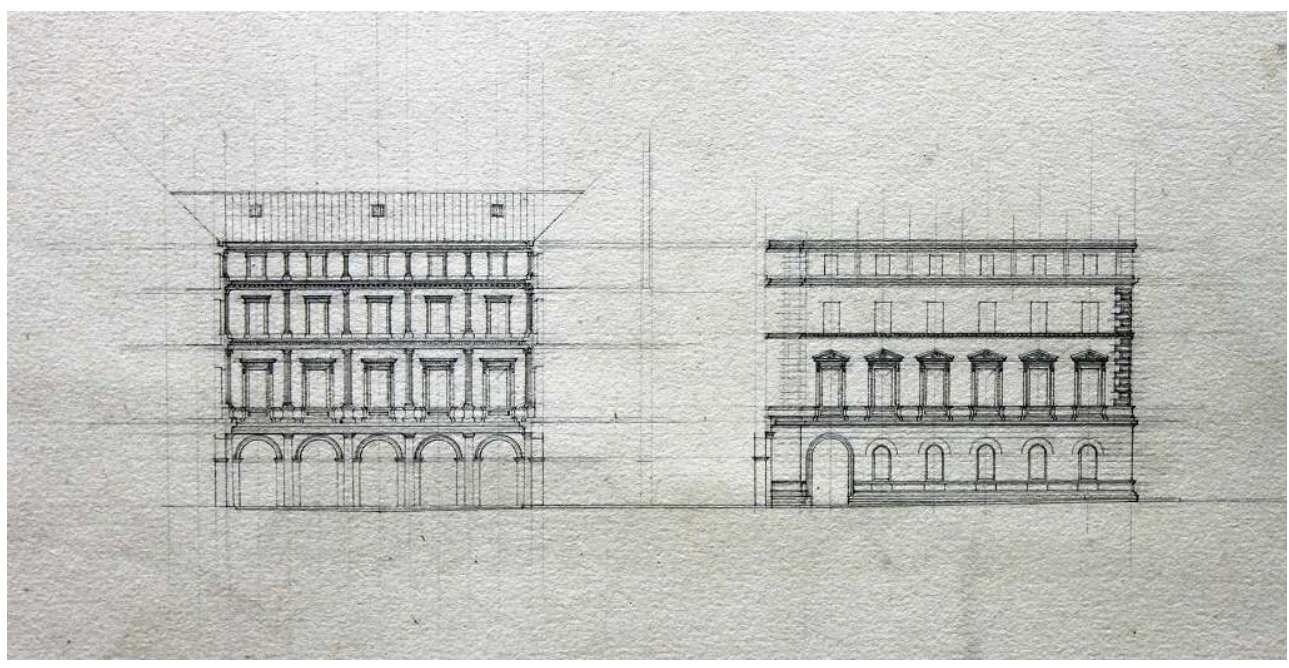

Deux études pour des élévations du bâtiment de la préfecture de police, quai des Orfèvres, Arthur Diet, dessins au crayon, vers 1860, conservé aux Archives de Paris (37Fi 91).

Reproduction Nicolas Courtin.

Entrepris en 1862, ce chantier aurait dû être la dernière étape de la constitution de la nouvelle cité judiciaire de la capitale. Les événements de 1871 modifièrent ce programme et entraînèrent une seconde vague d'importants travaux.

\section{Les reconstructions après l'incendie de 1871}

27 À l'instar du palais des Tuileries et de l'Hôtel de Ville, le palais de justice subit un incendie le 24 mai 1871. L'ensemble du bâtiment est touché mais les degrés variables de destruction vont appeler des réponses architecturales différentes. Formulées en août 1871, elles sont mises en œuvre jusqu'en 1895, date à laquelle un nouveau plan de répartition des locaux est dressé entre la Ville, le département et l'État ${ }^{21}$. Sont partiellement touchés, sans nécessité de reconstruction du gros-œuvre, les bâtiments de la Cour de cassation, des Assises et de la police correctionnelle. Dans ce cas, il s'est principalement agi de réparation et de reconstruction des aménagements intérieurs.

Les dommages les plus lourds ont porté sur la salle des pas perdus et la Grand'chambre ainsi que sur les bâtiments de la Cour des comptes et celui de l'instruction. Pour ces deux derniers, les dommages sont tels que le parti de la démolition totale a été retenu. Ainsi, à l'emplacement du bâtiment élevé par Jacques V Gabriel en 1738-1740, Duc et Dommey conçoivent-ils les nouveaux locaux de la cour d'appel. Dans une organisation assez complexe, ils s'étendent de la cour de la Sainte-Chapelle jusqu'au revers de la galerie Mercière tout en intégrant les vestiges du bâtiment dit «Renaissance » situés entre ces deux zones. La conception et la réalisation de cette partie du palais a connu une chronologie complexe, avec de nombreuses évolutions du projet dont les derniers plans sont donnés en 1894 par Honoré Daumet (1826-1911), l'architecte de la cour d'appel depuis 1879. C'est à partir de ce chantier que le fonds conserve des séries complètes de dessins techniques, attachements de maçonnerie principalement, qui renseignent utilement sur les structures actuelles. Outre les relevés concernant le bâtiment de Gabriel, cette partie du fonds est particulièrement intéressante pour les dessins consacrés à la décoration intérieure - notamment celle de la Première chambre, 
où se déploie un grand décor d'inspiration Renaissance. Parallèlement, la salle des pas perdus fait l'objet d'une quasi-reconstruction, alors que la salle basse doit être consolidée ${ }^{22}$. Les relevés effectués après l'incendie font état de l'importance des dégâts sur les maçonneries anciennes déjà plusieurs fois restaurées [fig. 10]. À cette occasion, les deux voûtes en berceau sont reconstruites, et les nouveaux locaux de l'État civil sont aménagés au-dessus.

\section{Figure 10}

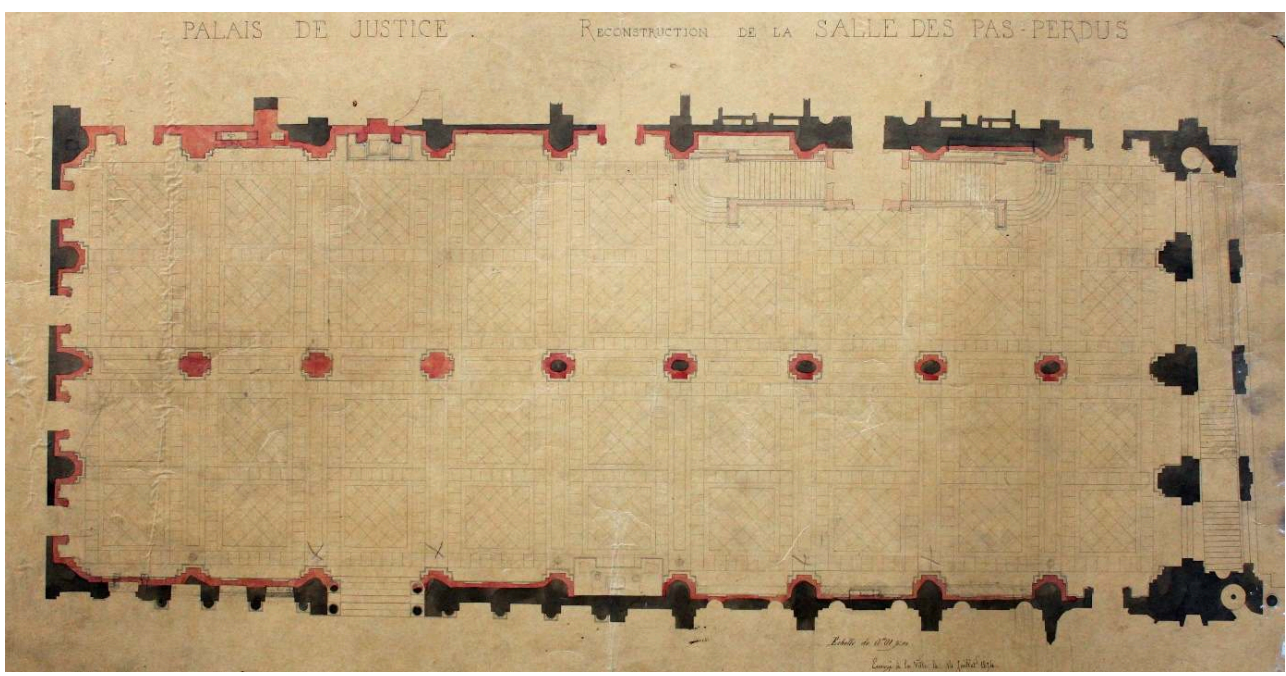

Palais de justice. Reconstruction de la salle des pas-perdus, anonyme, dessin, encre et lavis de couleurs, 1874, conservé aux Archives de Paris (37Fi 41).

Reproduction Nicolas Courtin.

La Grand'chambre attenante à la salle, ancien cœur du palais royal et salle d'audience principale du tribunal civil, a été dévastée. Sa restauration, entreprise en 1876, offre dans notre fonds une série importante de dessins d'architecture intérieure, décoration, ameublement, etc. [fig.11]. 
Figure 11

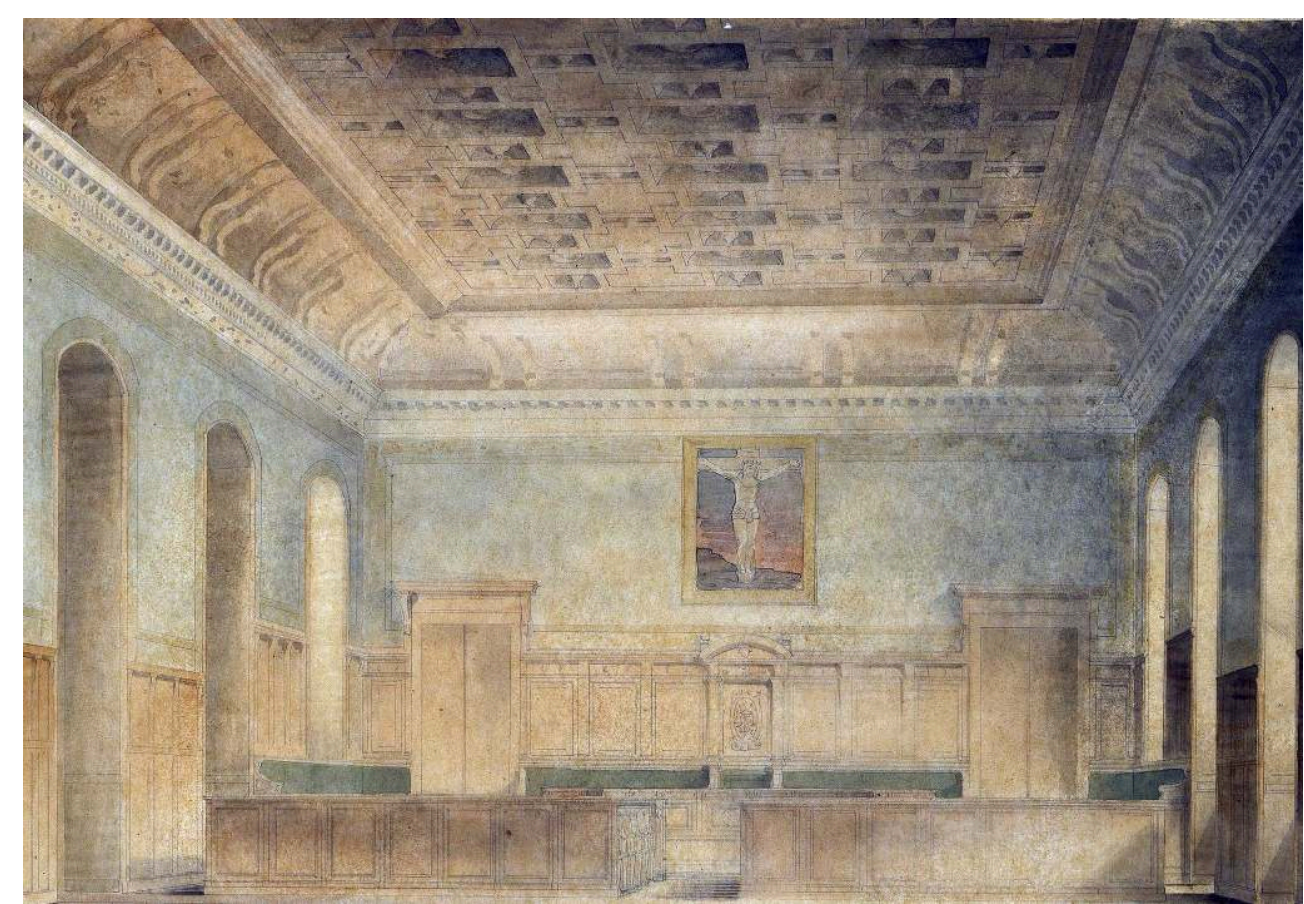

Projet pour la reconstruction de la grande chambre du tribunal civil de la Seine, anonyme, dessin aquarellé sur calque, vers 1860-1880, conservé aux Archives de Paris (37Fi 220).

Reproduction Nicolas Courtin.

Ces pièces forment un partie du corpus plus important constitué par les études et dessins d'exécution des décors des différentes juridictions dont la presque totalité des aménagements intérieurs est réalisée sous la III ${ }^{\mathrm{e}}$ République. Ainsi, le bâtiment des Assises et du dépôt est-il achevé en 1875 et les derniers travaux de décoration de la Cour de cassation sont livrés en 1892 (inauguration de la Grand'chambre). À travers ces études d'aménagements intérieurs se dessinent les hésitations des différents architectes, et notamment d'Honoré Daumet, qui succède à Duc comme architecte du tribunal de la Seine et de la cour d'appel. C'est sans doute à lui que l'on doit l'homogénéité des décors des principales salles d'audience pour lesquels il a généralement opté pour une inspiration baroque [fig. 12]. 
Figure 12

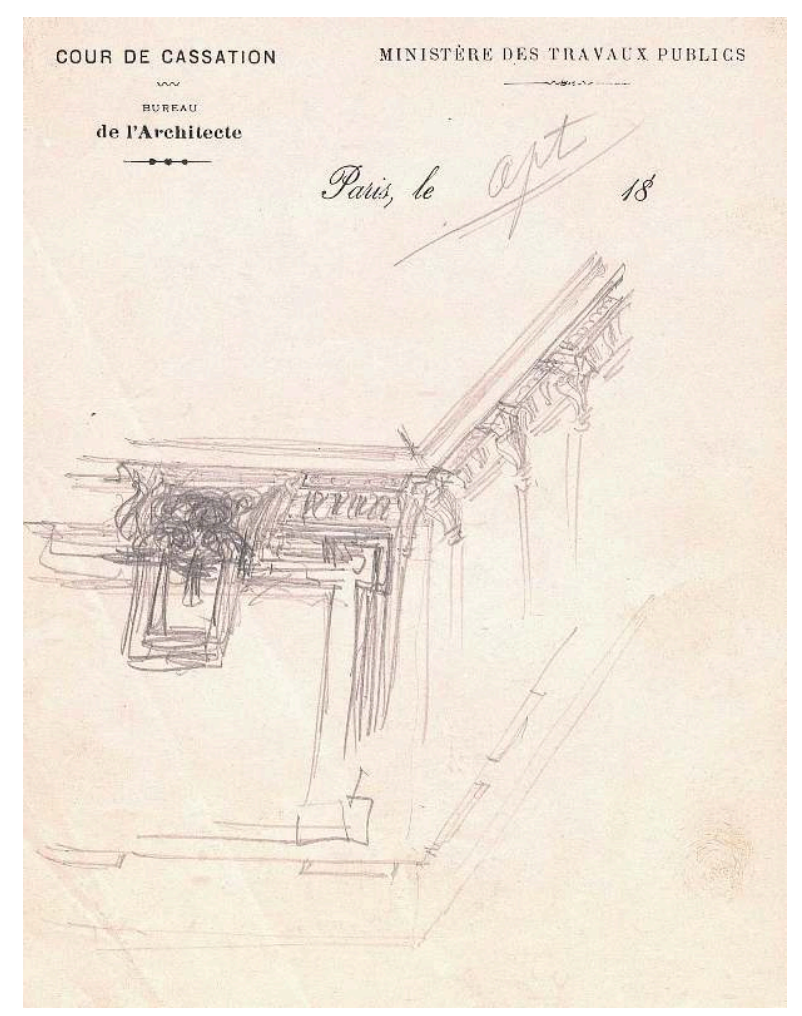

Croquis pour un élément de décor intérieur, architecte de la Cour de cassation, crayon, vers 1870-1880 (37Fi 79).

Reproduction Nicolas Courtin.

La dernière étape de l'évolution du palais de justice a été « l'agrandissement du tribunal de la Seine » conduit par l'architecte Albert Tournaire (1862-1958). Il s'est agi de créer un bâtiment neuf à l'angle du boulevard du Palais et du quai des Orfèvres - afin d'accueillir le tribunal correctionnel. Ce nouvel édifice entraînait la disparition des derniers immeubles environnant le Palais et devait livrer une nouvelle façade vers la rive gauche, en prolongement de celle de Guillaume et Diet. Tournaire se distingua en favorisant une élévation d'inspiration Renaissance française sur le quai et classique sur le boulevard. Les dessins de ce chantier sont particulièrement nombreux dans le fonds des Archives de Paris. Ils permettent de suivre très précisément toutes les étapes de la conception et de la construction de ce bâtiment neuf, dont les premiers projets sont datés de mai 1904, et qui fut livré en 1914. Après cette dernière construction, les documents conservés illustrent des travaux d'entretien ou d'aménagements intérieurs jusque dans les années 1960 [fig. 13], reflétant - principalement pour la cour d'appel et la Cour de cassation, une perpétuelle recherche de place et de nouveaux locaux. 
Figure 13

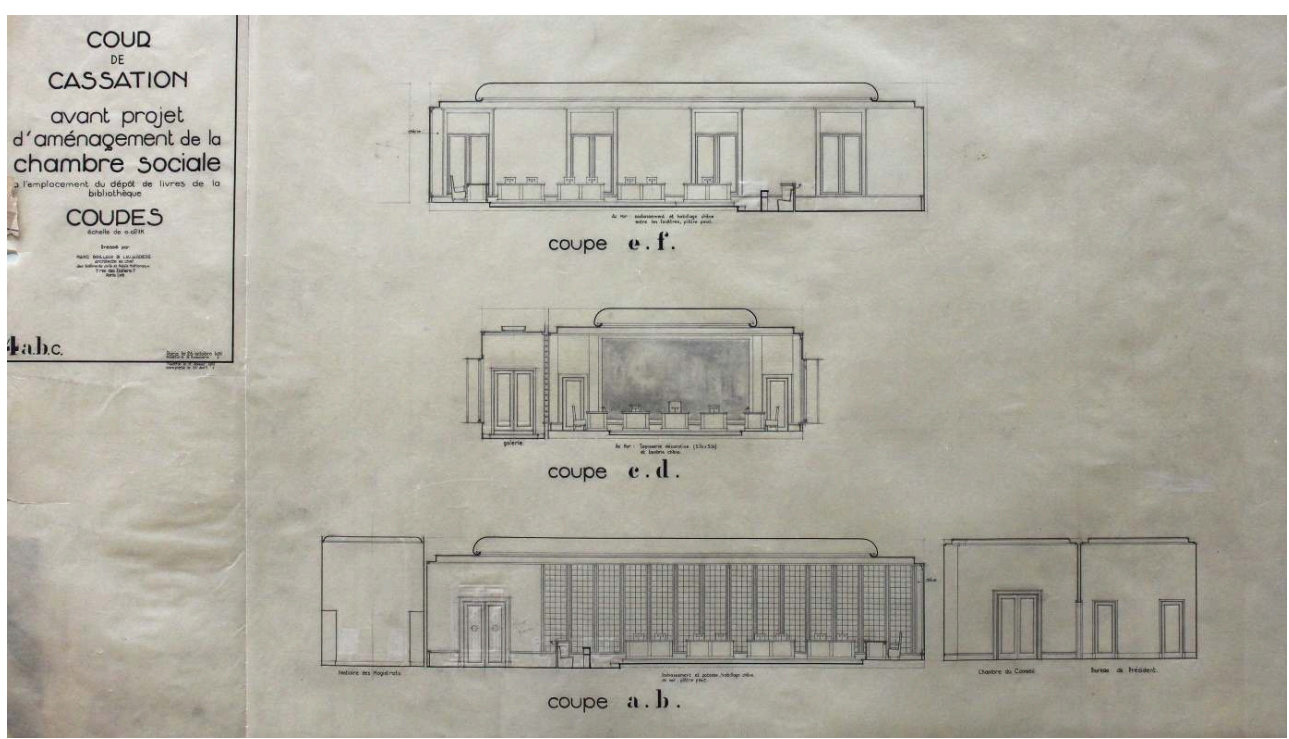

Cour de cassation. Avant-projet d'aménagement de la chambre sociale [...]. Coupes, Marc Brillaud de Laujardière, 1951-1952, conservé aux Archives de Paris (37Fi 59).

Reproduction Nicolas Courtin.

Ainsi, à la veille du premier conflit mondial, le projet d'isolement et d'agrandissement du palais initié par Guyot dans les années 1835-1840 trouva-t-il son accomplissement. Naturellement, la mise en œuvre s'est beaucoup écartée du projet de la Restauration. Comme d'autres bâtiments civils et palais nationaux parisiens, son histoire architecturale reflète les moments de l'histoire et sa destruction en 1871 témoigne de la permanence de sa considérable force symbolique - mais aussi de la permanente capacité à se reconstruire. Les dessins conservés par les Archives de Paris illustrent, par l'alternance des relevés et des projets, ces métamorphoses continues. Ils permettent aussi de mieux comprendre le fonctionnement d'une agence d'architecture d'un palais national, d'envisager les hésitations des architectes entre les partis à suivre : les styles à donner à l'architecture de la justice et de l'incarcération et la place de la modernité dans un édifice historique.

\section{Observations et pistes de réflexion}

La particularité du fonds conservé aux Archives de Paris est d'être exclusivement graphique. Les documents sont totalement indépendants des pièces écritesmajoritairement conservées aux Archives nationales dans les séries consacrées au conseil des Bâtiments civils. Un certain nombre de marques - cachets, signatures ou autres - relient certains dessins à la procédure de validation par le Conseil ${ }^{23}$. Mais la nature administrative particulière des locaux fait apparaître dans certains cas un autre système de visa, notamment un enregistrement préfectoral. On peut ainsi retracer le complexe parcours administratif du processus de création d'architecture publique.

Ces mentions sur les dessins sont précieuses pour la chronologie des projets et l'identification de leurs auteurs. Si Duc et Dommey ont été particulièrement attachés à signer leurs documents - à la main ou d'un cachet humide à leurs initiales -, les autres architectes ont rarement apposé leur nom sur les feuilles. C'est naturellement le cas des 
dessins les plus précoces dans le processus créatif comme les nombreux croquis du bâtiment du tribunal correctionnel, qu'il convient d'attribuer à Tournaire.

Ces feuilles identifiées permettent de dresser un cadre de classement des projets et d'imaginer la succession des phases du chantier ainsi que d'identifier les architectes responsables. Mais la majorité du fonds consiste en des feuilles anonymes - documents de travail qui n'ont pas de valeur réglementaire ou contractuelle, produits dans l'anonymat de l'agence.

Bon nombre de dessins du fonds portent des mentions d'envoi ou de remise à des entrepreneurs ou des artisans - apportant parfois une confusion dans l'auteur du dessin lui-même. Il s'agit de modèles - de sculpture, de structure, de mobilier, etc. délivrés par l'agence aux personnes chargées de les mettre en œuvre. La date à laquelle le dessin a été confié est parfois indiquée, précisant ainsi la chronologie des travaux. La variété des dessins reflète les différentes étapes de la création architecturale, de la conception d'ensemble au dessin d'exécution à l'échelle 1 du décor ou du mobilier. La chaîne complète du travail de l'agence peut être reconstituée dans bien des cas notamment pour la cour d'appel ou le bâtiment de Tournaire - avec son lot de dessins très proches, faux doubles et duplicatas. En termes de conservation, cette variété pose de véritables problèmes de cohérence des fonds: un même projet peut être conçu, préparé et diffusé sur des supports différents, à l'aide de techniques également variées.

Associées à la genèse du projet, les pièces documentaires sont rassemblées pour former la culture visuelle, les sources d'inspiration et les connaissances de l'architecte et de son agence. Il peut s'agir de dessins extérieurs, de gravures, de photographies...

Ce dernier support apparaît également employé par l'agence d'architecture pour documenter les travaux effectués - que ce soit les parties du bâtiment à démolir (logis $\mathrm{du}$ roi par exemple) ou les constructions neuves (vestibule de Harlay, notamment ${ }^{24}$ ). Exceptionnellement, le dessin aquarellé a été préféré afin de rendre la polychromie de décors découverts lors de démolitions autour de la Grande Salle ${ }^{25}$. Le travail continu des architectes autour d'un ensemble de bâtiments médiévaux et de l'époque moderne a formé leur propre goût et l'on peut formuler l'hypothèse que les parties anciennes du Palais sont suffisamment emblématiques pour avoir constitué la source principale d'inspiration des créateurs de la cité judiciaire du XIX ${ }^{e}$ siècle. Le sujet en soi mériterait une investigation plus poussée. Il convient toutefois de remarquer l'importance des relevés de détails ou de parties de bâtiments anciens, conservés ou non. La chronologie rapide des travaux a montré des attitudes variées quant au style retenu pour les constructions neuves. La Cour de cassation a démontré sa volonté d'indépendance d'esprit en ne se référant à aucune partie du Palais ancien. Duc et Dommey ont prouvé leur capacité à créer une architecture d'accompagnement tout autant qu'une architecture résolument moderne. Au final, le Palais du XIX ${ }^{e}$ siècle - bien que quasiment entièrement reconstruit - se présente toujours comme un collage d'architectures diverses et non pas comme un édifice organique unique - une anthologie de l'architecture française du Moyen Âge à la Belle Époque.

40 Cette constatation attire l'attention sur le style de l'architecture de la justice - qu'il conviendrait également d'approfondir spécifiquement pour le cas du Palais à la lumière des travaux de Caroline Soppelsa ${ }^{26}$. Dès les campagnes de Couture l'Aîné et Pierre Desmaisons pour la nouvelle entrée du Palais sous Louis XVI, la question s'est posée. En déplorant l'absence de caractère répressif de la nouvelle architecture, Louis-Sébastien 
Mercier soulignait déjà le problème de l'impression que devaient produire les bâtiments de la justice sur les justiciables ${ }^{27}$. Force est de constater que les architectes $\mathrm{du} \mathrm{XIX}^{\mathrm{e}}$ siècle au Palais de Paris n'ont pas véritablement répondu à la question - à l'exception peut-être du vestibule de Harlay.

À l'intérieur, les décors de la III $^{\mathrm{e}}$ République font étalage d'un luxe historicisant appelant allégories et motifs habituels à la rescousse d'un discours convenu. Werner Szambien ${ }^{28}$ a rappelé l'importance dans l'émergence du goût néogothique français des chantiers de restitution de décors entrepris par Duc et Dommey dans les parties médiévales - dont témoignent principalement les projets pour la tour de l'Horloge ${ }^{29}$ [fig. 14].

\section{Figure 14}

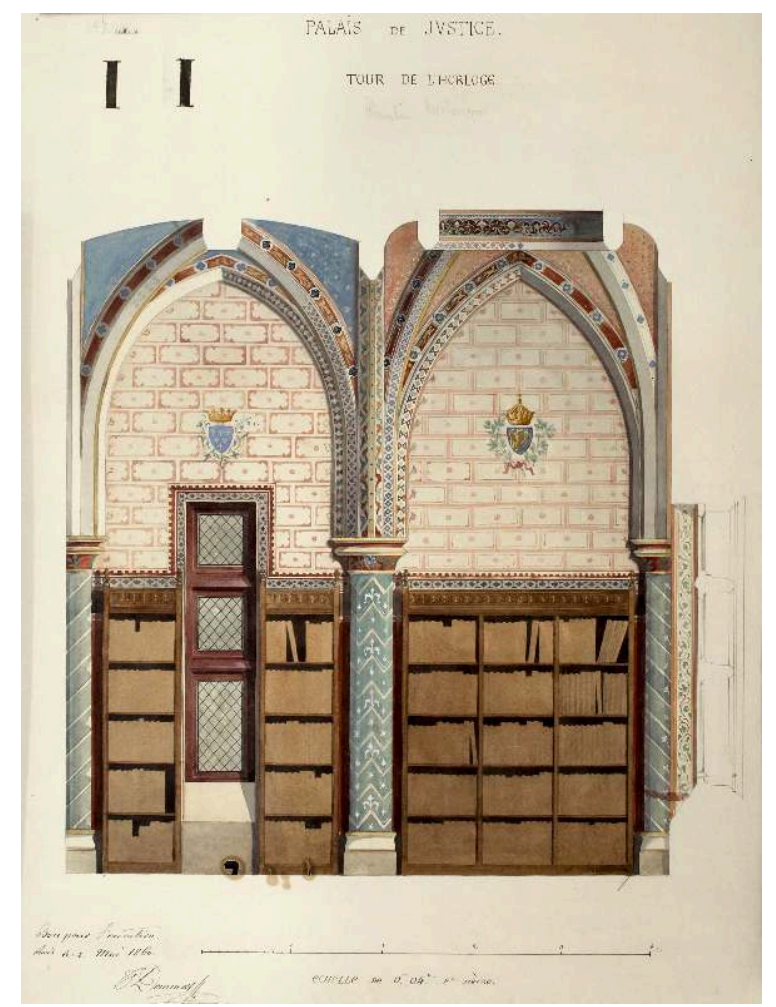

Palais de justice. Tour de l'Horloge. Chambre historique, Théodore Dommey, dessin aquarellé, 1860, conservé aux Archives de Paris (37Fi 165).

Reproduction Nicolas Courtin.

On retiendra en outre, disséminés dans les différents portefeuilles, de nombreux dessins relatifs à la spécificité des lieux - principalement le dépôt - qui mettent en lumière l'originalité de ce bâtiment, à la fois palais et prison. Aménagement de cellules, dessin de grilles, de portes fortes, de mobilier adapté, mise en place de sanitaires pour les prisonniers [fig. 15]... le fonds est riche d'informations de détail sur le second œuvre carcéral qu'il conviendrait d'étudier plus avant.

Les architectes du Palais ont dû en permanence, du projet initial à son achèvement en passant par sa réparation, effectuer des choix entre le processus de conservation/ restauration et celui de démolition / reconstruction. À ce titre, le Palais est un terrain d'exercice permanent de la jeune pratique de la conservation du patrimoine - et donc à la pointe des réflexions contemporaines sur l'architecture. 
Figure 15

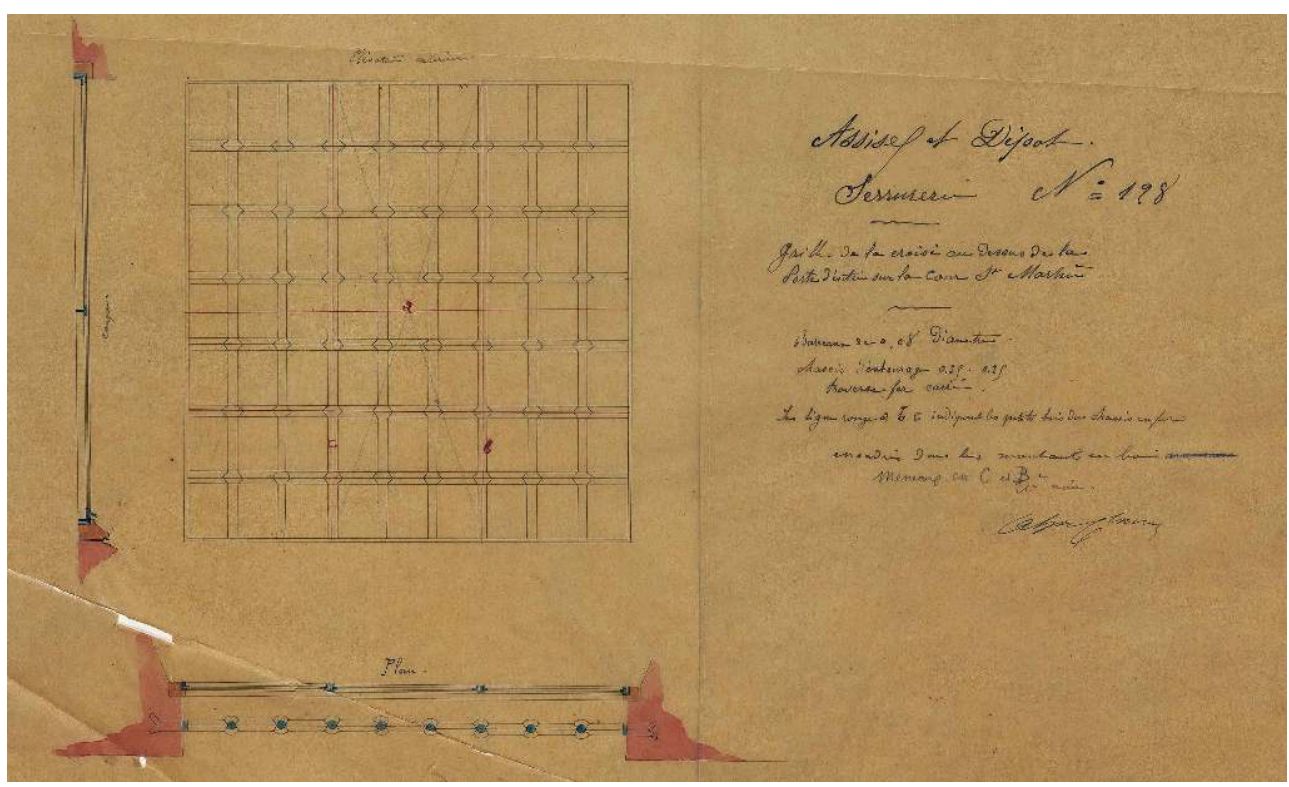

Assises et Dépôt. Serrurerie. Grille de la croisée au-dessus de la porte d'entrée sur la cour Saint-Martin, anonyme, encre noire sur calque, 1861-1866, conservé aux Archives de Paris (37Fi 180).

Reproduction Nicolas Courtin.

Les différents choix effectués par les architectes présentés plus haut trouvent de multiples justifications - intellectuelles, factuelles, économiques, topographiques... Mais qu'il s'agisse de restauration ou de construction nouvelle, la question de la modernité est omniprésente. Les dessins des architectes témoignent du recours régulier aux techniques les plus avancées. Le cas des voûtes de la Grande Salle est à ce titre emblématique, tout comme le recours abondant aux charpentes métalliques [fig. 16] - y compris lors de la restauration des bâtiments gothiques - puis l'emploi précoce du béton armé par Tournaire. 
Figure 16

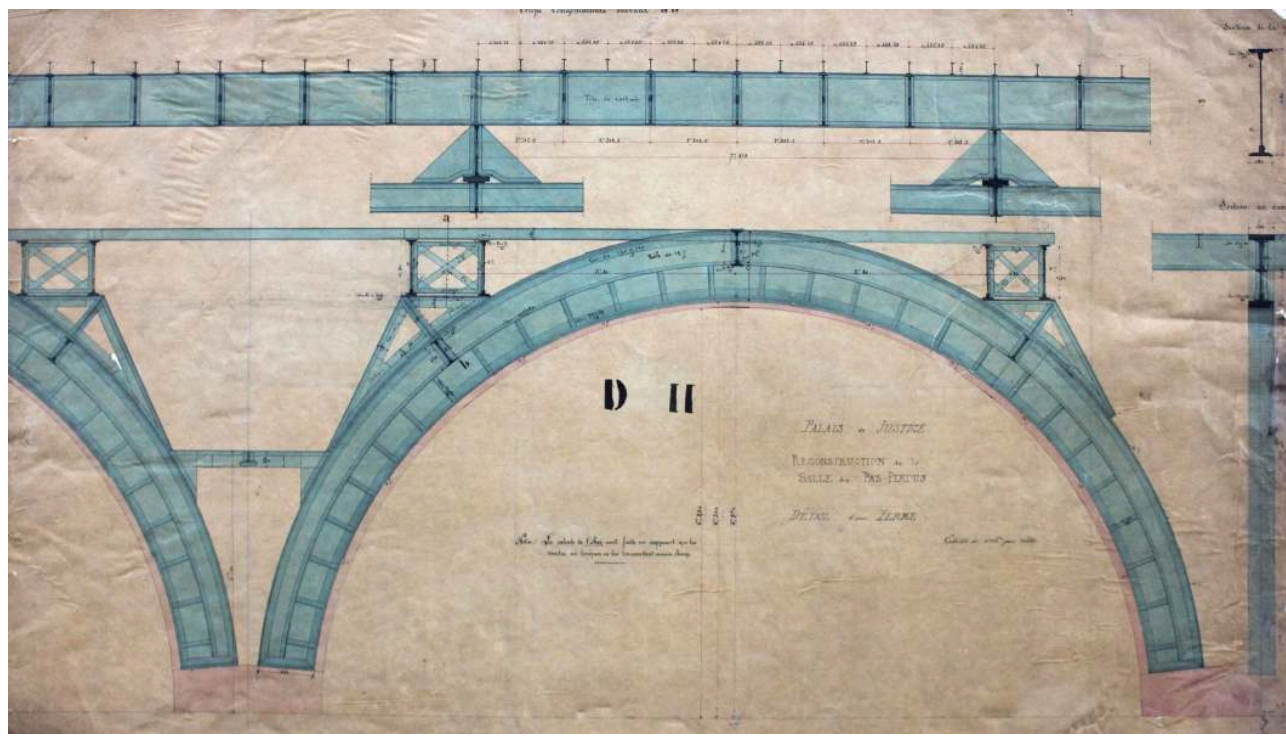

Palais de justice. Reconstruction de la salle des pas-perdus. Détail d'une ferme, anonyme, encre et lavis de couleurs sur calque, vers 1850-1870, conservé aux Archives de Paris (37Fi 38).

Reproduction Nicolas Courtin.

On trouvera également dans les nombreuses pièces concernant la mise en place de systèmes de chauffage ou de ventilation le recours aux techniques les plus modernes mises en œuvre dans des bâtiments neufs mais aussi patrimoniaux. Mais dans tous les cas, se rencontre la «main légère et pieuse » évoquée à propos des travaux d'Honoré Daumet sur les monuments historiques ${ }^{30}$.

Le fonds de dessins d'architecture 37Fi des Archives de Paris est un trésor méconnu. Il peut être abordé par de multiples points d'entrée. Mais sa richesse est aussi sa faiblesse: pléthorique, souvent muet, parfois en mauvais état et difficilement manipulable, il n'est compréhensible qu'à la lumière de sources conservées dans d'autres institutions. La récente mise à disposition du cadre de classement de ce fonds est une invitation à l'adresse de la curiosité de tous les chercheurs.

\section{NOTES}

1. SZAMBIEN Werner \& TALENTI Simona, Étude historique et patrimoniale du Palais de justice de Paris (schéma directeur, lot $n^{\circ} 4$ ). Rapport réalisé à l'issue de la deuxième phase, Paris, Institut parisien de recherche Art, Histoire, Architecture, littérature Développement, 1995.

2. GROUPE DE RECHERCHE ART HISTOIRE ARCHITECTURE LITTÉRATURE, Le Palais de justice de

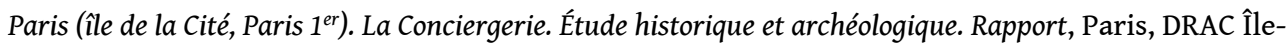
de-France / Conservation régionale des monuments historiques, 1997.

3. THOMASSIN Luc, TALENTI Simona, OZANAM Yves, ROBERT Hervé \& SZAMBIEN Werner (dir.), Le Palais de justice de Paris, catalogue d'exposition (exposition organisée à la Conciergerie), Paris, Action artistique de la Ville de Paris, coll. « Paris et son patrimoine », 2002. 
4. Voir http://archives.paris.fr/r/154/un-palais-de-justice-pour-paris/ [lien valide en novembre 2021].

5. Voir http://archives.paris.fr/arkotheque/inventaires/ead_ir_consult.php? $a=4 \& r e f=F R A D 075 \_003767$ [lien valide en novembre 2021].

6. Planche $\mathrm{n}^{\circ} 11 \mathrm{du}$ recueil des vingt vues de Venise gravées par Jean-Baptiste Brustoloni (ou Brustolon, 1712-1796) d'après Antoine Canaletto, [Venise], Lodovico Furlanetto, 1763.

7. DOMENC Paul, «Cour d'Appel de Paris, escalier de la $25^{\mathrm{e}}$ chambre », 2 mai 1966 (37Fi 59). Voir lien note 5 .

8. Par commodité, le classement du fonds suit une description par secteurs de l'édifice, englobant parfois plusieurs services. On distingue ainsi cinq secteurs principaux : le tribunal de la Seine (secteur au nord et au sud de la cour du Mai), le tribunal correctionnel (partie orientale du quai des Orfèvres), la préfecture et la cour d'appel (partie occidentale du quai des Orfèvres), la cour d'assises et le dépôt (secteur oriental du Palais, vers la place Dauphine) et la Cour de cassation (partie orientale du quai de l'Horloge).

9. 37Fi 316.

10. 37Fi 320.

11. 37Fi 318.

12. Documents conservés dans les fonds des Archives nationales et de la Médiathèque de l'architecture et du patrimoine.

13. À titre indicatif, nous avons établi la succession des architectes titulaires comme suit :

Tribunal de la Seine : Jean-Nicolas Huyot (1780-1840) : 1835-1840; Louis Duc (1802-1879) (avec Théodore Dommey) : 1840-1879; Théodore Dommey (1801-1872) (avec Louis Duc) : 1840-1871; Honoré Daumet (1826-1911) (avec Louis Duc): 1871-1911; Albert Tournaire (1862-1958): 1911-1958; Jean Hulot (1871-1959): entre 1914 et 1937; Marc Brillaud de Laujardière (1889-1973) : avant 1944 et après 1962.

Cour de cassation : Louis Lenormand (1801-1862) : 1849-1862 ; Louis Duc (1802-1879) : 1862-1879 ; Ernest Coquart (1831-1902) : 1879-1890 ; Paul Domenc (1906-1979) : 1954-1970.

Cour d'appel : Honoré Daumet (1826-1911) : 1879-1911 ; Paul Domenc (1906-1979) : 1954-1970.

Préfecture de police : Émile Gilbert (1793-1874) : 1855-1869 ; Arthur Diet (1825-1890) : 1869-1890 ; Gabriel Midy (1873-1955) : vers 1924-1926.

14. SZAMBIEN Werner \& TALENTI Simona, Étude historique et patrimoniale du Palais de justice de Paris (schéma directeur, lot $n^{\circ} 4$ ). Rapport réalisé à l'issue de la deuxième phase.

15. On se reportera au plan coté $4003 \mathrm{~W} 8934$ pour le plan masse du projet Huyot ou bien aux Archives nationales.

16. GASTALDI Nadine, «L'architecture publique au XIX ${ }^{\mathrm{e}}$ siècle. Richesse et variété des sources des Archives nationales ", Livraisons d'histoire de l'architecture, $\mathrm{n}^{\circ} 21,2011$, disponible en ligne https:// journals.openedition.org/lha/267 [lien valide en novembre 2021]. Ainsi qu'aux Archives nationales : sous-série $\mathrm{F} / 21$.

17. État des constructions et délimitations en 1838. Premier étage (37Fi 1).

18. Pour l'organisation ancienne du palais, voir les restitutions proposées in DELHUMEAU Herveline, Le Palais de la Cité. Du palais des rois de France au Palais de Justice, Paris / Arles, Cité de l'architecture et du patrimoine / Actes Sud, coll. « Les grands témoins de l'architecture », 2011.

19. DUC Louis Duc \& DOMMEY Théodore, Projet d'isolement et d'agrandissement du palais de justice.

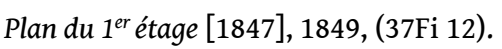

20. Sur la question du rapport des architectes du XIX siècle aux parties anciennes du Palais, voir : "Le XIX ${ }^{\mathrm{e}}$ siècle face au passé. Regarder et transformer l'architecture des XVII et XVIII ${ }^{\mathrm{e}}$ siècles ", communication à la journée d'étude éponyme (Paris, Centre André Chastel, 11 septembre 2019). Hélas, à part le programme disponible via http://www.centrechastel.paris-sorbonne.fr/ actualites/le-xixe-siecle-face-au-passe-0 [lien valide en novembre 2021], je ne crois pas qu'il y ait la moindre trace pour l'instant de cette journée d'étude sur le site du Centre. 
21. 37Fi 3.

22. 37Fi 41.

23. Sur ce processus, voir GASTALDI Nadine, "L'architecture publique au XIX ${ }^{\mathrm{e}}$ siècle. Richesse et variété des sources des Archives nationales ».

24. 37Fi 22.

25. 37Fi 45.

26. SOPPELSA Caroline, « Le XIX ${ }^{\mathrm{e}}$ siècle et la question pénitentiaire : un siècle d'expérimentations architecturales dans les prisons de Paris ", thèse de doctorat en histoire de l'art sous la direction de Jean-Baptiste Minnaert (École doctorale Sciences de l'homme et de la société), Tours, université de Tours, 2016.

27. Cité dans COURTIN Nicolas, Paris au XVIII siècle. Entre fantaisie rocaille et renouveau classique, Paris, Parigramme, 2013, p. 59.

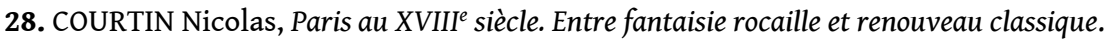

29. 37Fi 165.

30. ROUJON Henry, Notice sur la vie et les travaux de M. Honoré Daumet (1826-1911) : lue dans la séance publique annuelle du 9 novembre 1912, Paris, Institut de France, Académie des beaux-arts, 1912, p. 15, disponible en ligne https://gallica.bnf.fr/ark:/12148/bpt6k64568554.texteImage [lien valide en novembre 2021].

\section{RÉSUMÉS}

Les Archives de Paris conservent, sous la cote 37Fi, le fonds des pièces graphiques de l'ancienne agence d'architecture du palais de justice de Paris. Son classement récent a permis de constituer un instrument de recherche détaillé et d'en rendre possible la consultation.

Cet ensemble remarquable permet de suivre les réflexions et les réalisations des architectes ayant la charge du Palais entre 1838 et 1915 principalement - soit les temps de création de la cité judiciaire qui a occupé les lieux jusqu'en 2018, de son détachement complet du palais d'Ancien Régime aux derniers agrandissements à la veille du premier conflit mondial. La richesse de ce fonds provient aussi bien des informations sur l'histoire architecturale de l'édifice que sur la manière de travailler d'une agence d'architecture des Bâtiments civils au XIX ${ }^{\mathrm{e}}$ siècle.

En revenant sur la chronologie de travaux que ces dessins précisent, nous nous sommes attaché à pointer les particularités de ce fonds de dessins d'architecture: la méthode de travail des agences, les problématiques techniques, l'illustration des pratiques de conservation et de restauration des bâtiments anciens ou la recherche d'une esthétique de la justice.

The Archives of Paris preserve, under section 37Fi, the collection of architect's drawings of the former architectural office of Paris's palais de justice (courthouse). Its recent classification has provided a detailed research instrument and has made its consultation possible.

As we reviewed the timeline of the works, specified by these drawings, we have strived to point out the distinctive characteristics of this collection of architectural drawings: working methods of the offices, technical issues, illustration of conservation and restoration practices of old buildings or the quest for an aesthetics of justice. 


\section{INDEX}

Keywords : This outstanding group of documents allows to keep track of the thinking and achievements of the architects in charge of the courthouse, mainly between 1838 and 1915 - that is the creation time of the judicial precinct that occupied the premises until 2018, from its complete detachment from the palais of Ancien Regime to the last expansions on the eve of the First World War. The richness of this collection comes from both information on the architectural history of the building and on the way an architectura, the Civilian buildings used to work in the 19th century.

Mots-clés : justice, patrimoine, Restauration, reconstruction, tribunaux, mobilier, décor, dessins, métier d'architecte

\section{AUTEUR}

\section{NICOLAS COURTIN}

Docteur en histoire de l'art.

Chef du service de la conservation et des technologies numériques des Archives de Paris. nicolas.courtin@paris.fr 